\title{
Teaching motor skills by means of biomechanical analysis of the motion: the physiological basis and applied information technologies
}

\author{
A.V. Razuvanova ${ }^{1, a}$, E.V. Koshelskaya ${ }^{1,2}$, I.A. Karpova ${ }^{1}$, E.V. Medvedeva ${ }^{1}$ \\ ${ }^{1}$ Tomsk Polytechnic University, 634050 Lenin Avenue, 30, Tomsk, Russia \\ ${ }^{2}$ Tomsk Regional Teachers Professional Re-training Institute, 634034 Pirogova str., 10, Tomsk, Russia
}

\begin{abstract}
The article proves the possibility of training athletes using motor skills on the basis of biomechanical analysis of movements with application of information technologies. Motion Tracking digital single frame shooting photography - is proposed as a method for biomechanical analysis. The relevance of this method is conditioned by the results of the study of a repulsion phase in the performing of the standing jump by athletes of different qualifications. The conclusion about the importance of an optimal model of a jump based on biomechanical analysis is given, and the formation of athletes' skills, using information technologies and the principle of urgent information, is discussed.
\end{abstract}

\section{Introduction}

At the present stage of the development of physical culture and sports the importance of technically correct performance of any motion is still in the first place, as it is the main criterion for evaluation. The compliance with the standard technique of performing one or the other movement always has an impact on the result.

Since the introduction of modern information technologies happens everywhere and the critical opinion of judges is now protected from mistakes and supported by accurately reading programs, the smallest technical errors become visible and easily analyzed [13]. All this requires finding fundamentally new approaches to training athletes, based on the use of capabilities of modern technologies in the training process $[4,5]$.

There is no doubt that modern technologies provide a wide range of possibilities and allow receiving a huge amount of data, which should be correctly interpreted and applied $[6,7,10]$. Therefore, improvement of the technological side of the training process will be effective only if proposed solutions are based on fundamentally new approaches due to the profound physiological and biomechanical analysis.

Thus, in the present study we decided to apply a modern technological approach, based on biomechanical analysis of performing basic motor skills, for recognizing the mechanisms of their formation in qualified athletes and identifying opportunities to improve the effectiveness of training.
In order to do this it is necessary to evaluate by means of digital technology, to what extent the qualification of athletes is dependent on their physiological characteristics during performance of motor actions. We should identify in what way and by what adjustment of motion control systems for performing a motor action happens in athletes and develop recommendations for the mastering of motor actions with the application of information technologies.

Objective of study is to justify the possibility of teaching motor skills to athletes basing on the biomechanical analysis of movements with the use of information technology.

\section{Materials and methods}

A standing jump was selected as a motor action. This motor action is used as a preparation in the training process of athletes, as well as a part of the standard school programs for physical education. Long jump results from a technical viewpoint depend on the phase repulsion; it is the very phase that we have elected for analysis and implementation of the goal.

We have surveyed 30 athletes of the male sex between the ages of 17 and 24 years old. They were divided into two groups according to the degree of motor skill formation in standing jump.

The main study group included highly skilled athletes (16 people), who had been doing jumps for more than four years and specialize in speed-strength types of athletics. The control group included students of the

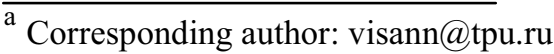


department of sports disciplines (14 persons), who were engaged in some other sports and did not have any sports categories in athletics.

For biomechanical analysis of functional systems, the Motion Tracking method - single frame shooting by a digital high-speed camera - was applied.

Recording equipment is Vision Camcorder Research Phantom Mire X2. Photographing was conducted at the speed of 100 frames per second. Research and Infographics were created in the program "StarTraceTracker 1.1 VideoMotion ${ }^{\circledR} "$.

\section{Results and discussion}

During the push off phase feet of the jumper appear to be in a static position. The main action is performed with hands - it is a swing, and the body is the lever for the swing. It looks exactly like this in the representatives of the control group, who perform repulsion motion, keeping a static position of the foot and pelvis. That is to say, they do squat (bend the knee joint) and produce the swing with arms, leaning their body forward.

\section{Description of the control group}

From the vertical position (the angle of the body $=180^{\circ}$ ) with arms bent in the elbow, they make an upward movement to their head. In the final point of lifting arms, the angle of the elbow is $\geq 70$ (figure $1, x=0.5$ ). The body is static at this moment.

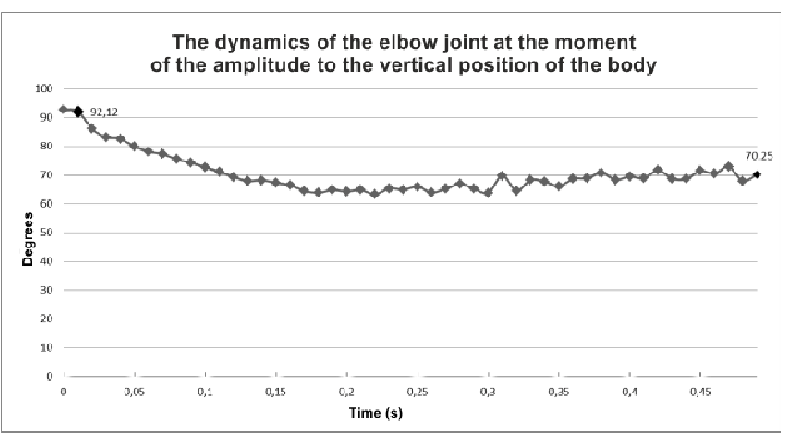

Fig. 1. A long jump, a repulsion phase of the control group

This is followed by a sharp lowering of folded hands. The body leans forward and a squat is performed with the knee joint.

At this point of transition from the vertical position to the squat, the jumper swings his arms down and extends his arms smoothly in the elbows to $170^{\circ}$ already behind his back. The slope of the body in the final point of the swing is $\geq 100 \circ$ (figure $2, x=1.4$ ).

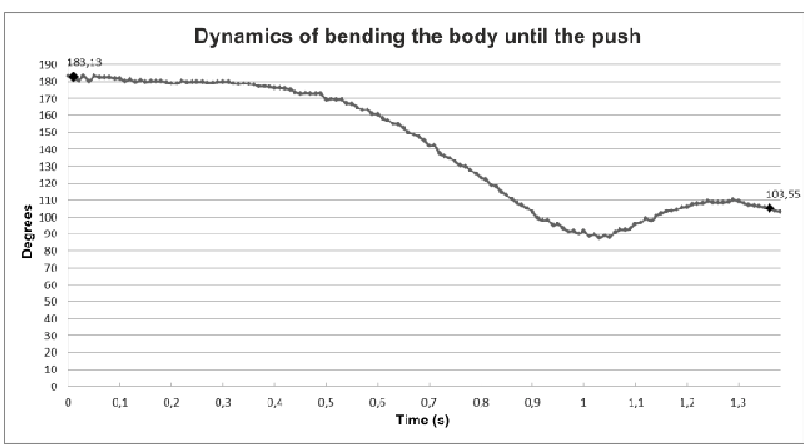

Fig. 2. A long jump, a repulsion phase of the control group

The key point of the repulsion is that a push follows this movement [12]. In the control group only at this point the foot begins to work, the heel is detached, that is, the weight is transferred to the forefoot. The push, as shown by the findings, is quite a typical action [13], and representatives of the main and the control group performed it fairly typically: they vigorously throw both arms forward and up, straighten diagonally forward to the direction of movements until the detachment of the legs and move to the unsupported position. The main difference in the push movement of the representatives of two groups is the angle of straightening the knee at the moment of detachment from the support area. It became clear that at this moment the representatives of the control group do not straighten the knee to the end, leaving the knees bent.

Thus, the angle of the knee at the time of detachment of feet from the support area is $\approx 150^{\circ}$ (figure $3, \mathrm{x}=$ $0.54)$.

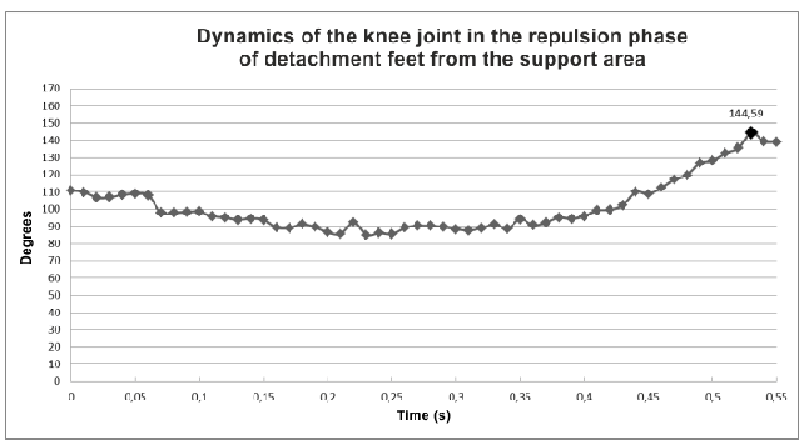

Fig. 3. A long jump, a repulsion phase of the control group

\section{Description of the main group}

The repulsion phase for professional athletes is of the greatest importance [14], since the length of the jump depends on all steps of this phase.

Analysis showed that the process of repulsion of the representatives of the main group is a more complicated coordinating movement compared to the control group. Firstly, the time of the phase itself in the main group covers $50-60 \%$ of the total time, while representatives of the control group spend only $\approx 15 \%$ of total time on it. Secondly, feet perform rolls during repulsion phase and actively influence on the overall motion of the body, but are not static, as it occurs in case of representatives of the control group [11]. 
Thirdly, the dynamics of movement angles and the amplitude of abduction and reduction are at times more, which is confirmed by the graphical analysis based on the method of Motion caption.

From the vertical position (the angle of the body $=$ $180^{\circ}$, figure $5, \mathrm{x}=0$ ) with arms straight in the elbow, sportsmen carry out the upward movement towards the head. At the final moment of lifting arms, the angle of the elbow joint is $\geq 150^{\circ}$ (figure $4, \mathrm{x}=0.8$ ).

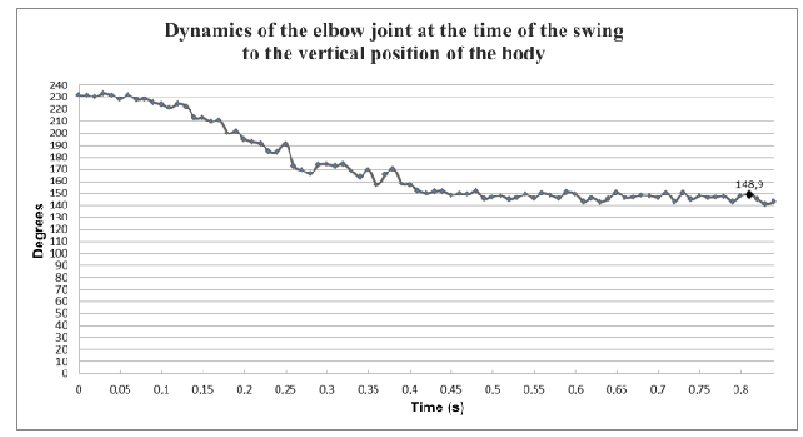

Fig. 4. A long jump, a repulsion phase of the main group

During performing this motion the body is not static, from the moment of the beginning of lifting arms to their vertical positions, body makes the deflection toward the jump, which is equal to $\approx 200^{\circ}$ (figure $5, \mathrm{x}=0.79$ ). That is, the jumper bends forward while rising straight arms upward, which we have not seen in the movements of the control group.

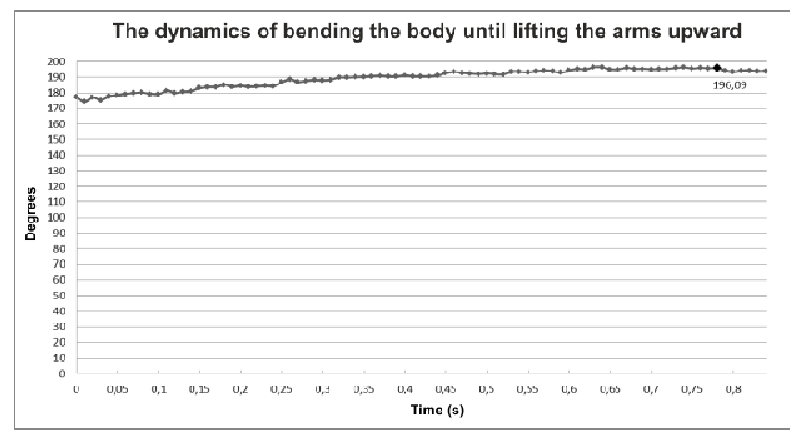

Fig. 5. A long jump, a repulsion phase of the main group

This is followed by a sharp lowering of straight arms, the body leans forward and a squat is performed, but now with the knee and ankle joints. As the foot actively works, there is a weight transfer of the body to the forefoot and heel comes off from the support area.

Squat is not deep, the working angle at the knee joint is $\approx 90^{\circ}$. At this point of transition from vertical position to the squat, athletes of the main group swing straight arms down and back. The slope of the body at the final point of the swing is $\approx 70^{\circ}$ (figure $6, \mathrm{x}=1.89$ ), which is much deeper than that of the control group.

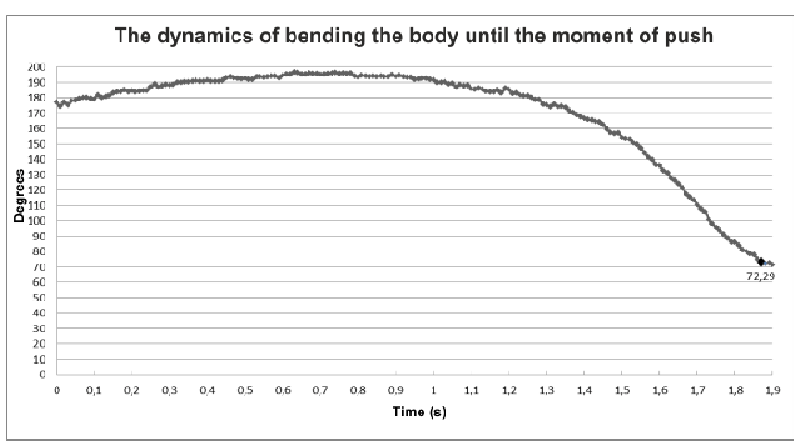

Fig. 6. A long jump, a repulsion phase of the main group.

The key point of the repulsion-push follows after it. As we have mentioned above, the push is quite a typical action for the representatives of both groups.

The main difference in the effect of the push in representatives of two groups is the angle of straightening the knee joint at the time of detaching feet from the support area. Thus, representatives of the main group straighten the knee completely, stretching all their body diagonally upward in the direction of the jump.

Thus, the knee joint angle at the time of detaching feet from support area is $\approx 180^{\circ}$ (figure $7, \mathrm{x}=0.54$ ).

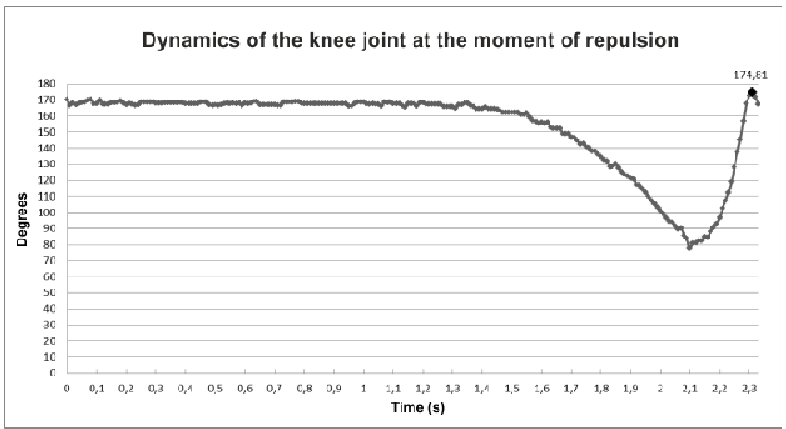

Fig. 7. A long jump, a repulsion phase of the main group

Due to the described parameters of the main group, that is performing the push off phase of action [12, 14], we can see that the motions of representatives of the control group are simplified and rather imitate the necessary motion technique without obtaining any actual results while performing the motion.

It is clearly confirmed by the visual difference in length of the jump between the representatives of two groups, but this is only the superficial view. By means of the Motion caption, the actual result influences the horizontal speed of the Common Center of the Body gravity point at the moment of transition to the phase of flight, the value of which is effective for the complete jump. According to this parameter, the difference between representatives of two groups is $\approx 1000 \mathrm{~mm} /$ second (figure 8). The speed of the Common Center of the Body gravity in representatives of the main group is $\approx 2200 \mathrm{~mm} / \mathrm{sec}$.

(Figure 8 , graph $\mathrm{B}, \mathrm{x}=2.3$ ). The speed of the Common Center of the Body gravity in representatives of the control group is $\approx 1200 \mathrm{~mm} / \mathrm{sec}$.

(Figure 8 , schedule $\mathrm{A}, \mathrm{x}=0.54$ ). If you pay attention to axis $x$ of the two graphs (figure 8), which reflects the 
duration of the repulsion phase, it is clearly evident that the representatives of the control group spend four-times less period to perform all the actions, but at the same time the speed of the Common Center of the Body gravity flight is considerably less.

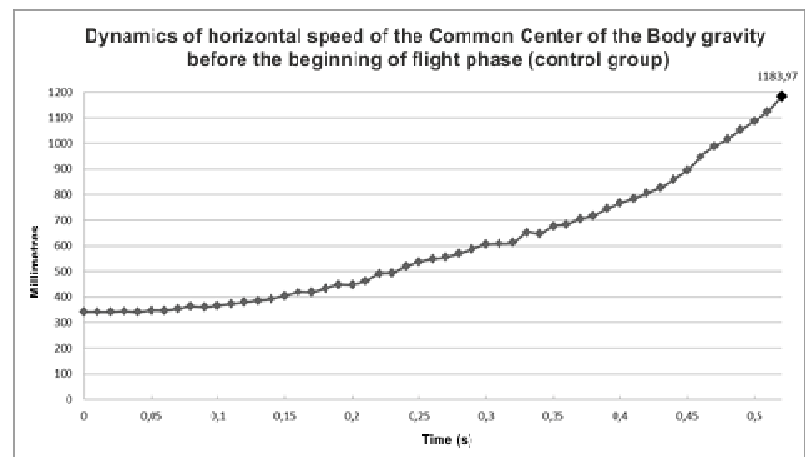

A

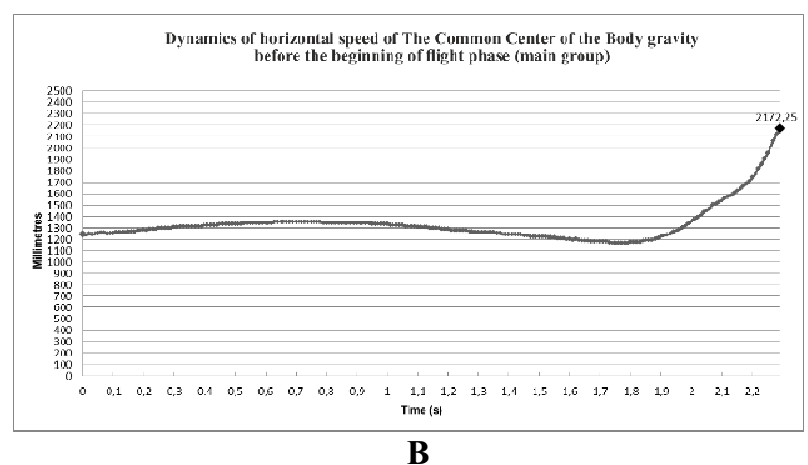

Fig. 8. Graphs of horizontal speed of The Common Center of the Body gravity point in the repulsion phase. A - a graph of the control group, B - a graph of the main group.

\section{Conclusion}

The results indicate that the mechanisms of the organization of standing jump motion for beginners and experienced athletes have qualitative differences. The beginners' jump is performed as a set of movements swing of the arms, a squat, a repulsion - only partially coordinated with each other.

At the same time, the athletes of the main group perform a standing jump as a single motion, all elements of which are strictly coordinated with each other and are performed in a certain sequence: powerful swing of the arms with simultaneous deflection of the body forward, then transfer of the body to the forefront of the foot, a squat down and withdrawal of the arms down with a simultaneous slope of the body - a set of potential energy for the jump. The result is a high flight speed and good outcome.

Apparently, the young athletes perform the long jump through the implementation of the independent motion stereotypes sets, which are poorly coordinated with one another.

During the training a set of independent movements is connected into a single motor stereotype with a consistent coordinated system of its elements. The development of such stereotype provides an optimal technique of performing motions and achievement of the best result $[8,9]$.

All mentioned above shows that the optimal training scheme of motions must be based not on learning separate elements, but on the formation of the general scheme of performing a jump.

We consider that the most reasonable approach is building an optimal model of the jump based on a biomechanical analysis and formation of the sportsmen's skill on the basis of application of information technologies using the principle of urgent information.

\section{References}

1. L.V. Kapilevich, E.V. Koshelskaya, S.G. Krivoschekov, HP, 41(4), (2015)

2. L.V. Kapilevich, L.V. Koshel'skaya, A.V. Razuvanova, TPFK, 7, (2015)

3. E.V. Koshelskaya, A.V. Razuvanova, O.S. Smerdova, L.V. Kapilevich, D.Yu. Balanev, TPFK, 12, (2014)

4. E.V. Koshelskaja, L.V. Kapilevich, V.N. Bajenov, V.I. Andreev, O.I. Buravel, BEBM, 2, (2012)

5. L.V. Kapilevich, TPFK, 7, (2012)

6. I.-M. Skavhaug, K.R. Lyons, A. Nemchuk, S.D. Muroff, S.S. Joshi, HMS, 47, (2016)

7. C.S. Thompson, S. Schabrun, P.W. Marshall, HMS, 47, (2016)

8. D.W. Powell, D.S.B. Williams, HMS, 44, (2015)

9. J.T. Cavanaugh, K.M. Guskiewicz, C. Giuliani, S. Marshall, V. Mercer, N. Stergiou, BJSM, 39 (11), (2005)

10. J.T. Cavanaugh, K.M. Guskiewicz, C. Giuliani, S. Marshall, V.S. Mercer, N. Stergiou, JAT, 41 (3), (2006)

11. W.T. Chen, Z.Z. Wang, X.M. Ren, JZU, 7 (10), (2006)

12. A.L. Aguinaldo, J. Buttermore, H. Chambers, JAB, 23 (1), (2007)

13. S. Dun, J. Loftice, G.S. Fleisig, D. Kingsley, J.R. Andrews, AJSM, 36 (4), (2008)

14. R.F. Escamilla, S.W. Barrentine, G.S. Fleisig, N. Zheng, Y. Takada, D. Kingsley, J.R. Andrews, AJSM, 35 (1), (2007) 